\title{
Simultaneously Synchrotron X-ray Fluorescence and Ptychographic Imaging of Frozen Biological Single Cells
}

\author{
S.Chen, ${ }^{1}$ J.Deng, ${ }^{2}$ Y.S.G.Nashed, ${ }^{3}$ Q.Jin, ${ }^{4}$ D.J.Vine,,${ }^{5}$ T.Peterka, ${ }^{3}$ K.Brister,${ }^{6}$ C.Jacobsen,,${ }^{1,2,4,7}$ S.Vogt ${ }^{1}$ \\ ${ }^{1}$ Advanced Photon Source, Argonne National Lab, Argonne, IL 60439, USA \\ ${ }^{2}$ Applied Physics, Northwestern University, Evanston, IL 60208, USA \\ ${ }^{3}$ Mathematics and Computing Science Division, Argonne National Lab, Argonne, IL 60439, USA \\ ${ }^{4}$ Department of Physics and Astronomy, Northwestern University, Evanston, IL 60208, USA \\ ${ }^{5}$ Advanced Light Source, Berkeley National Lab, Berkeley, CA 94720, USA \\ ${ }^{6}$ Synchrotron Research Center, Northwestern University, Argonne, IL 60439, USA \\ ${ }^{7}$ Chemistry of Life Processes Institute, Northwestern University, Evanston, IL 60208, USA
}

Bionanoprobe (BNP), a hard x-ray fluorescence sample-scanning nanoprobe at the Advanced Photon Source of Argonne National Laboratory, has been used to quantitatively study elemental distributions in biological cells with sub-100 $\mathrm{nm}$ spatial resolution and high sensitivity [1]. Cryogenic conditions enable biological samples to be studied in their frozen-hydrated state with both ultrastructure and elemental distributions more faithfully preserved compared to conventional chemical fixation or dehydration methods. Radiation damage is reduced in two ways: the diffusion rate of free radicals is decreased at low temperatures [2]; and the sample is embedded in vitrified ice, which reduces mass loss [3].

The BNP usually operates in an energy range of 4.5-25 keV. This energy range enables most elements of interest in biological systems to be studied by their K- or L-shell emission lines. For example, nuclear regions are generally high in $\mathrm{P}$ and $\mathrm{Zn}$ concentrations. Elevated $\mathrm{Mn}$ is often used to locate mitochondria. These interpretations can be enhanced if fluorescence maps are coupled with structural information from $\mathrm{H}, \mathrm{C}, \mathrm{N}$ and $\mathrm{O}$, the main constitutes of biological systems. Unfortunately, hard x-ray probes are nearly blind to these light elements due to their low fluorescence yields [4] and little absorption contrast at multi-keV x-ray energies.

Phase imaging techniques including Zernike phase contrast [5] and differential phase contrast [6] have been implemented to hard x-ray scanning probes to provide structural information. An alternative method is ptychography, a coherent diffraction imaging method. A sample is scanned through a coherent illumination with significant overlap. Far-field diffraction patterns are collected using a pixelated area detector. The diffraction patterns are then used to reconstruct the transmission function of the object via a phase retrieval method, yielding phase contrast images. One example demonstrating the applications of ptychography to biological sciences is by Vine et al [7]. The advantages of ptychography over other phase imaging methods are twofold: enhanced contrast and improved spatial resolution. In theory, the spatial resolution of ptychography is only limited by the wavelength of the incident x-ray photons.

We have recently demonstrated ptychographic imaging at the BNP [8] (Fig.1). The structural information of frozen biological single cells has been obtained in conjunction with elemental information (Fig.2). Concerns raised on the speed of both data collection and reconstruction particularly for potential 3D imaging. To improve the throughput, multi-probe modes [9] have been implemented in the reconstruction algorithm to address partially coherent illumination (higher flux, allowing shorter dwell time per pixel) [8] and fly-scan data collection (lower overhead time) [10]. Computation has been carried out using multiple GPUs with a hybrid parallel strategy [11]. 
[1] S Chen et al, J Synchrotron Rad 21 (2014), p. 66-75.

[2] M.C.R. Symons, Ultramicroscopy 10 (1982), p. 97-104.

[3] J Maser et al, J Microsc 197 (2000), p. 68-79.

[4] M Krause, J Phys Chem Ref Data 8(2) (1979), p. 307-27.

[5] C Holzner et al, Nature Physics 6 (2010), p. 883-7.

[6] MD de Jonge et al, PRL 100 (2008), p. 163902-1-4.

[7] DJ Vine et al, Opt Express 20(16) (2012), p. 18287-96.

[8] J Deng et al, PNAS 112(8) (2015), p. 2314-9.

[9] P Thibault et al, Nature 494(7435) (2013), p. 68-71.

[10] J Deng et al, Opt Express 23(5) (2015), p. 5438-51.

[11] Y Nashed et al, Opt Express 22(26) (2014), p. 32082-97.

[12] S Chen et al, Proc of SPIE 9592 (2015), p. 959201-1-6.

[13] The BNP is funded by NIH/National Center for Research Resources High End Instrumentation Grant 1S10RR029272-01. Ptychography implementation is supported by NIH/National Institute of General Medical Sciences under Grant 1R01GM104530. This research used resources of the Advanced Photon Source, a U.S. Department of Energy (DOE) Office of Science User Facility operated for the DOE Office of Science by Argonne National Laboratory under Contract No. DE-AC02-06CH11357.

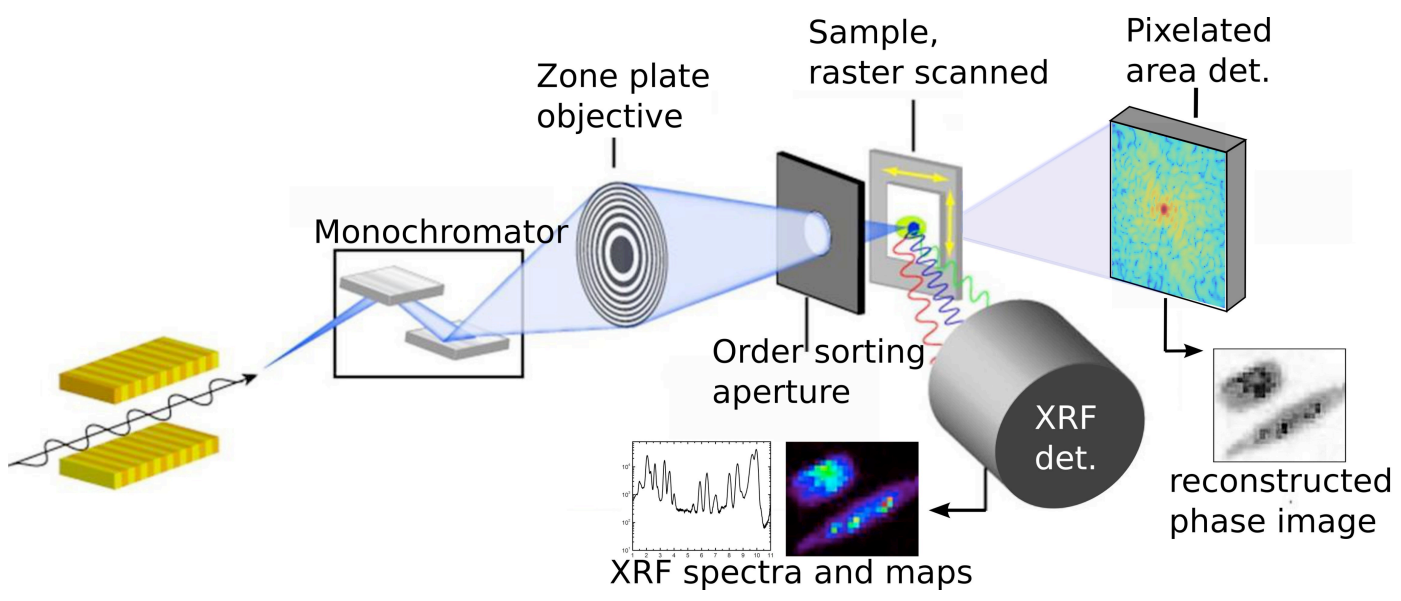

Figure 1. Schematic showing the experimental setup at the BNP. X-ray fluorescence spectra and farfield diffraction patterns are recorded, respectively, using a fluorescence detector at $90^{\circ}$ with regards to the incident beam and a pixelated area detector downstream of the sample [12].

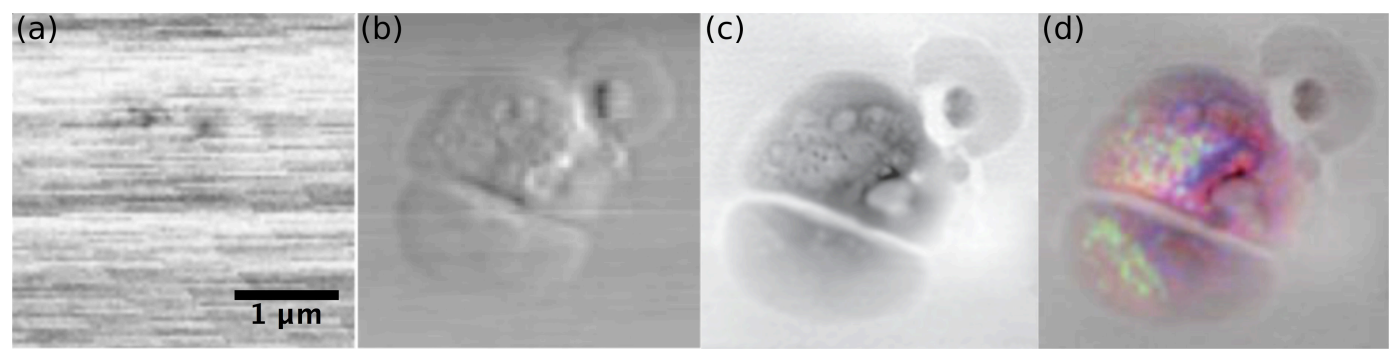

Figure 2. Comparison between (a) transmission contrast (b) differential phase contrast, and (c) ptychography image of a green algae cell, showing improved contrast and spatial resolution in (c). (d) Overlay of ptychography and $\mathrm{K}(\mathrm{red}), \mathrm{S}($ blue), and $\mathrm{P}$ (green) x-ray fluorescence images. 